\title{
SBML and Synthetic Biology
}

\author{
Chris J. Myers \\ University of Utah \\ COMBINE 2011 \\ September 5, 2011
}




\section{Motivation}

\section{HOW STANDARDS PROLIFERATE:}

(SEE: A/C GHRRGERS, GHARACIRR ENCOONINS, IN STANT MESSAGING, ETC)

SITUATION: THERE ARE 14 COMPETING STANDARDS.
14?! RIDICULOUS! WE NEED TO DEVELOP ONE UNIVERSAL STANDARD THAT COVERS EVERYONE'S USE CASES.
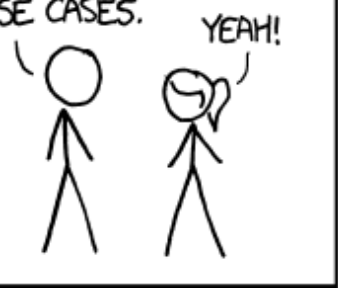

500N:

SITUATION: THERE ARE 15 COMPETING STANDARDS.

$\ldots$

๑ Standardized Model Description Language for Multi-Cellular Simulations:

등

- Several workshops including one last week at ICSB.

- Synthetic Biology Open Language (SBOL):

- Emerging standard for synthetic biology. 


\section{Motivation}

\section{HOW STANDARDS PROLIFERATE:}

(SER: A/C GHRRGERS, GHARAGIRR ENCODNGS, IN STANT MESSAGING, ETC)

SITUATION: THERE ARE 14 COMPETING STANDARDS.
14?! RIDICULOUS! WE NEED TO DEVELOP ONE UNIVERSAL STANDARD THAT COVERS EVERYONE'S USE CASES.
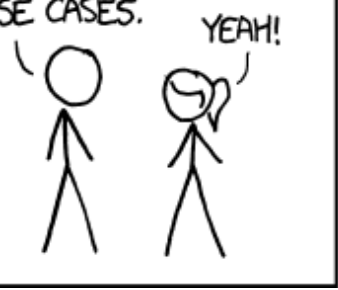

500N:

SITUATION: THERE ARE 15 COMPETING STANDARDS.

.

๑ Standardized Model Description Language for Multi-Cellular Simulations:

- Several workshops including one last week at ICSB.

- Synthetic Biology Open Language (SBOL):

ญ

- Emerging standard for synthetic biology. 


\section{Synthetic Biology Open Language (SBOL)}

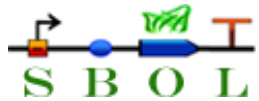

- Developed by the Synthetic Biology Data Exchange Working Group (SynBioDEX) including $>30$ members from academia and industry.

- SBOL meetings:

\begin{tabular}{|l|l|l|}
\hline April 2008 & 1st SynBioDEX Workshop & University of Washington \\
\hline July 2009 & 2nd SynBioDEX Workshop & Stanford University \\
\hline June 2010 & 3rd SynBioDEX Workshop & Anaheim, CA \\
\hline January 2011 & 4th SynBioDEX Workshop & VirginiaTech \\
\hline June 2011 & 5th SynBioDEX Workshop & San Diego, CA \\
\hline January 2012 & 6th SynBioDEX Workshop & University of Washington \\
\hline
\end{tabular}




\section{Recent Announcement}

ARPA-BAA-11-60: Living Foundries: Advanced Tools and Capabilities for Generalizable Platforms (ATCG):

กิ

To encourage interoperability, all applicable design tools and

databases developed under the ATCG program should be compatible with Synthetic Biology Open Language (SBOL) core data model. 


\section{Example SBOL Use Cases}

- Send a design template for it to be filled in with a DNA sequence.

Send an annotated DNA sequence for assembly planning.

- Publish a collection of DNA components to be re-used for design of novel DNA circuits.

- Retrieve and send annotated plasmid DNA sequence information between multiple software packages. 


\section{SBOL Core Data Model}
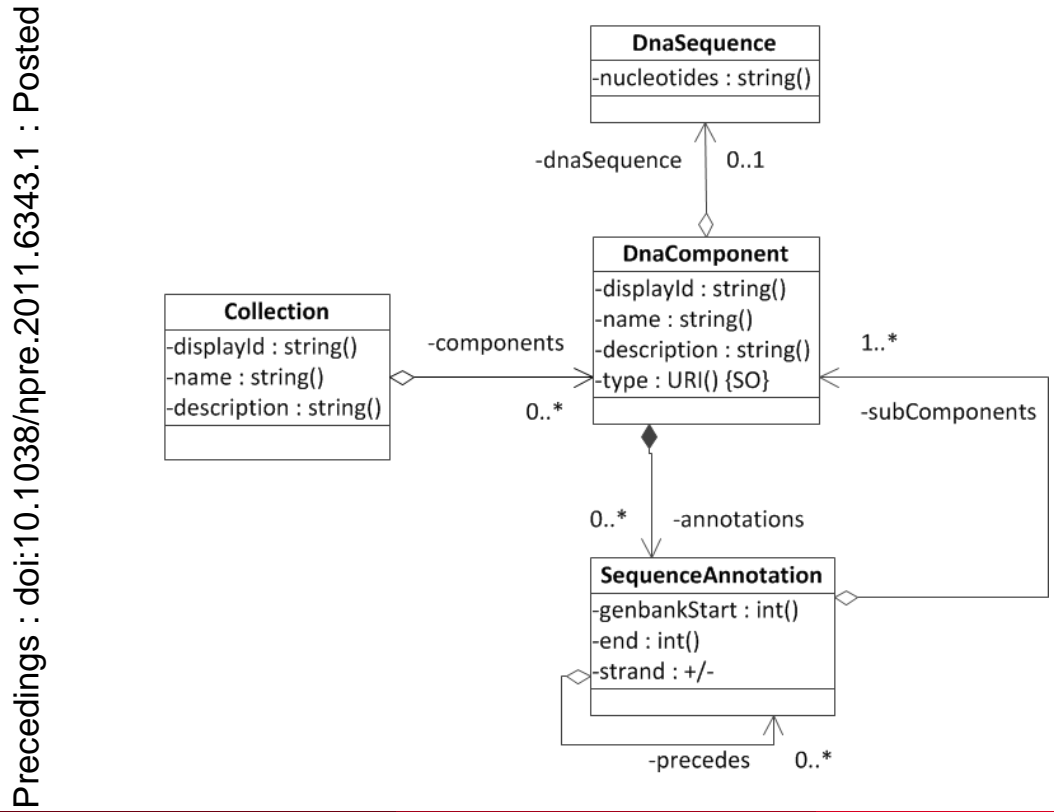


\section{Software Tools}

- libSBOLj - java implementation of SBOL core data model, will support serialization in RDF and XML.

- iBioSim - Chris Myers (University of Utah)

- TInkerCell - Herbert Sauro (University of Washington)

- Clotho - Doug Densmore (Boston University)

- GenoCAD - Jean Peccoud (VirginiaTech Bioinformatics Institute)

- gd-ice - Timothy Ham (Joint Bio Energy Institute)

- Electronic Datasheets - Cesar Rodriguez (BioFab) 


\section{SBOL in iBioSim}

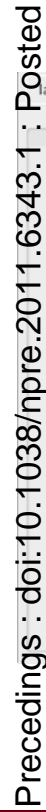




\section{SBOL in iBioSim}

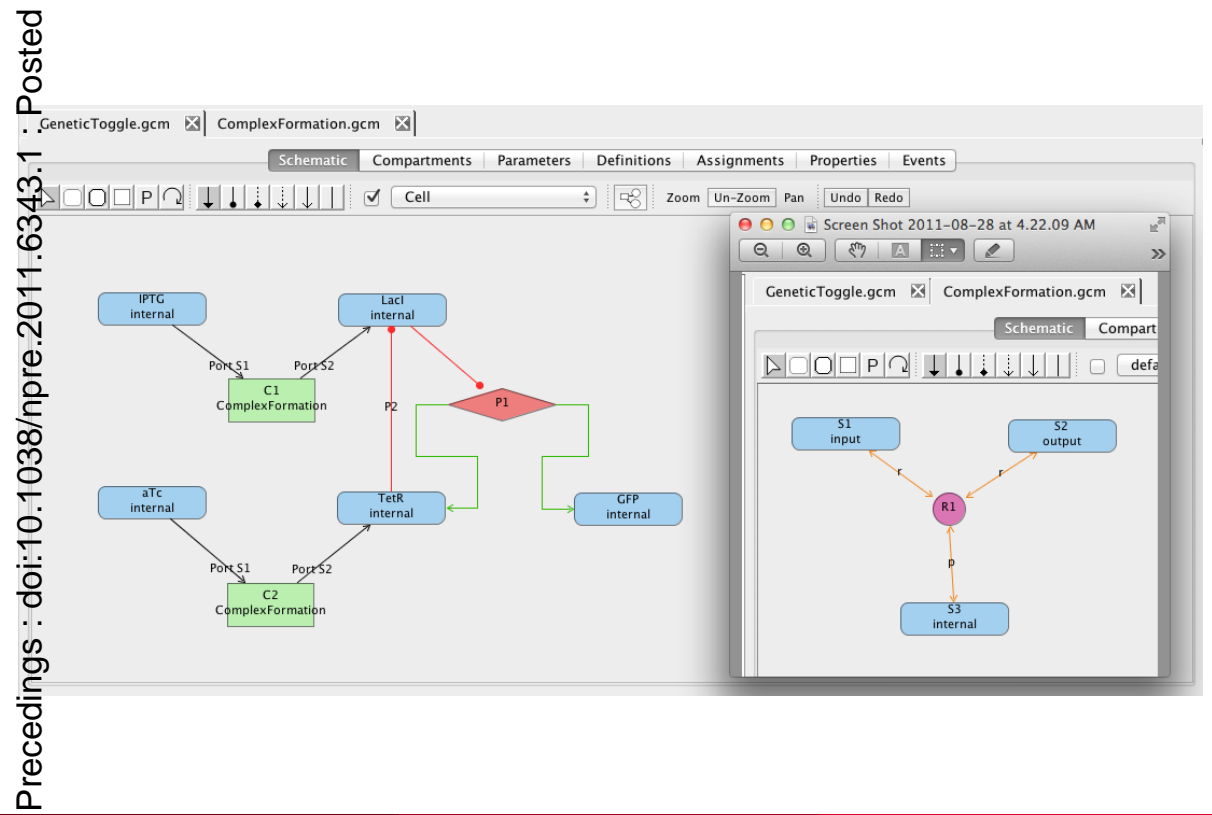




\section{SBOL in iBioSim}

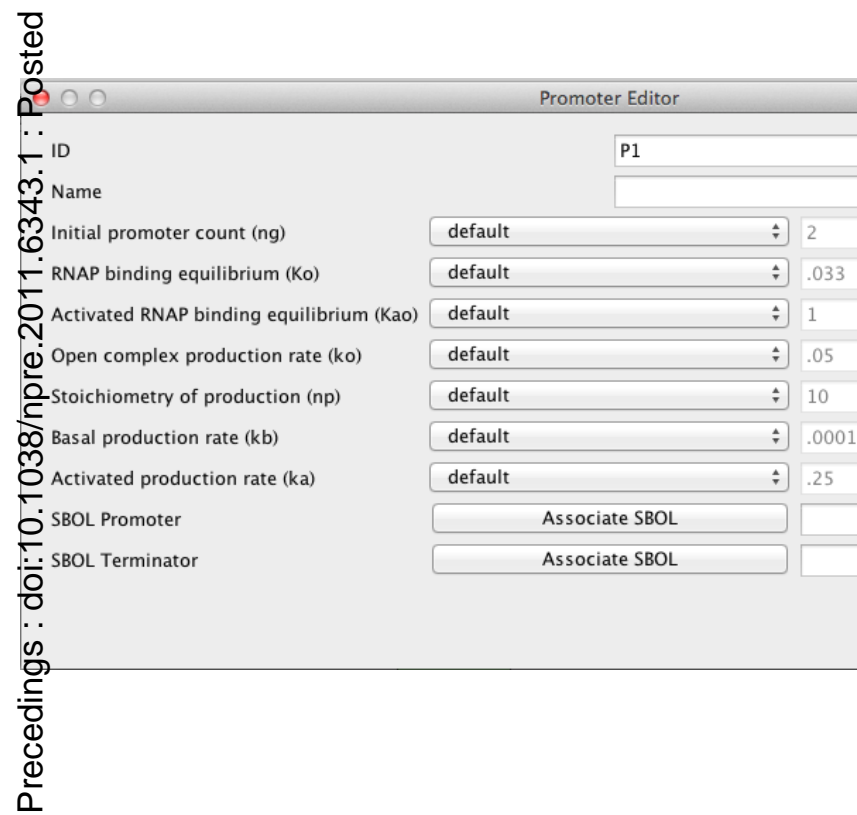




\section{SBOL in iBioSim}

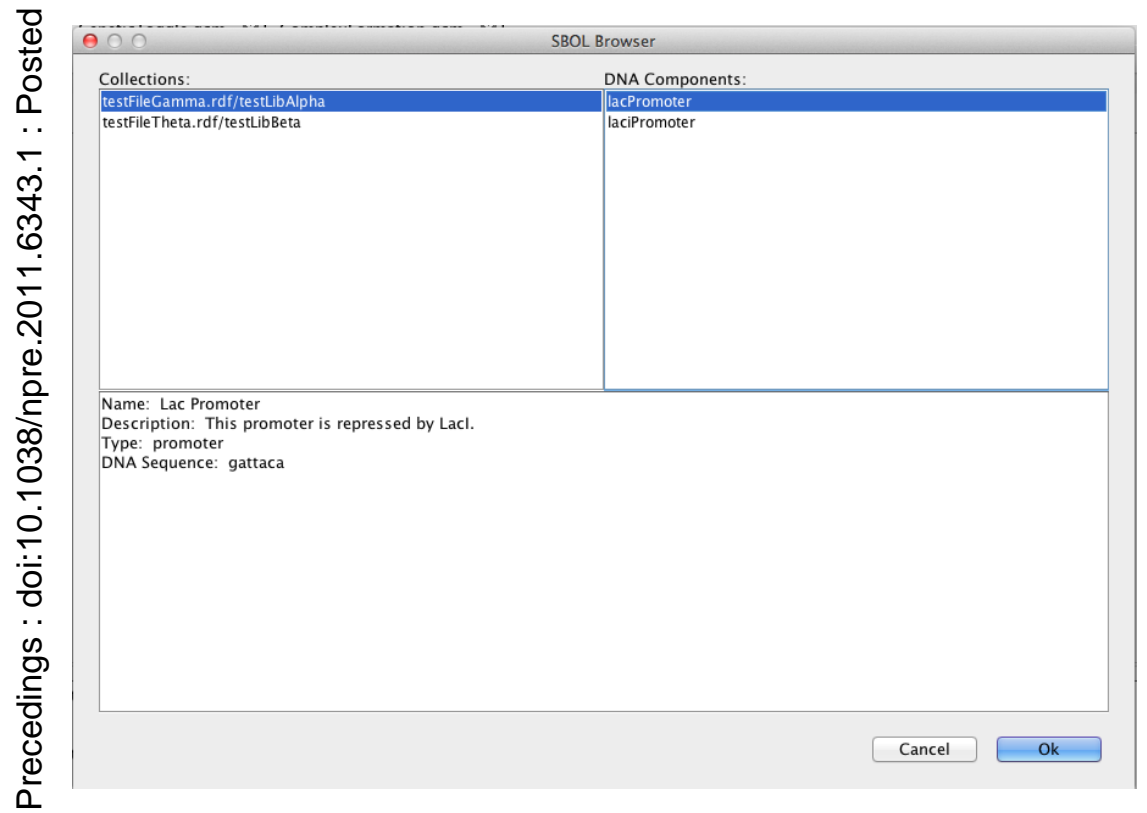




\section{SBOL in iBioSim}

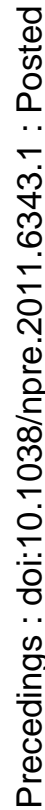

QP 1 Species Editor

ID

Name

Type

Compartment

Boundary Condition

Constant

Has Only Substance Units

Units

Conversion Factor

Initial amount/concentration (ns)

Degradation rate $(\mathrm{kd})$

Extracellular degradation rate (kecd)

Complex formation equilibrium (Kc)

Membrane diffusion rate ( $\mathrm{fd} / \mathrm{rv}$ ) ( $\mathrm{kmdiff}$ )

Extracellular diffusion rate (kecdiff)

SBOL Ribosome Binding Site

SBOL Open Reading Frame

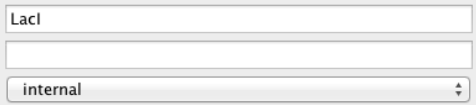

$\square$ diffusible $\square$ constitutive

\begin{tabular}{|l|}
\hline default \\
\hline false
\end{tabular}

\begin{tabular}{|c|c|c|}
\hline default & $\div$ & 0 \\
\hline default & $\div$ & .0075 \\
\hline default & $\div$ & .005 \\
\hline default & $\div$ & 0.05 \\
\hline default & $\div$ & $1.0 / 0.01$ \\
\hline default & $\leftarrow$ & 1.0 \\
\hline \multicolumn{2}{|c|}{ Associate SBOL } & \\
\hline & & \\
\hline
\end{tabular}

Cancel 


\section{SBOL in iBioSim}

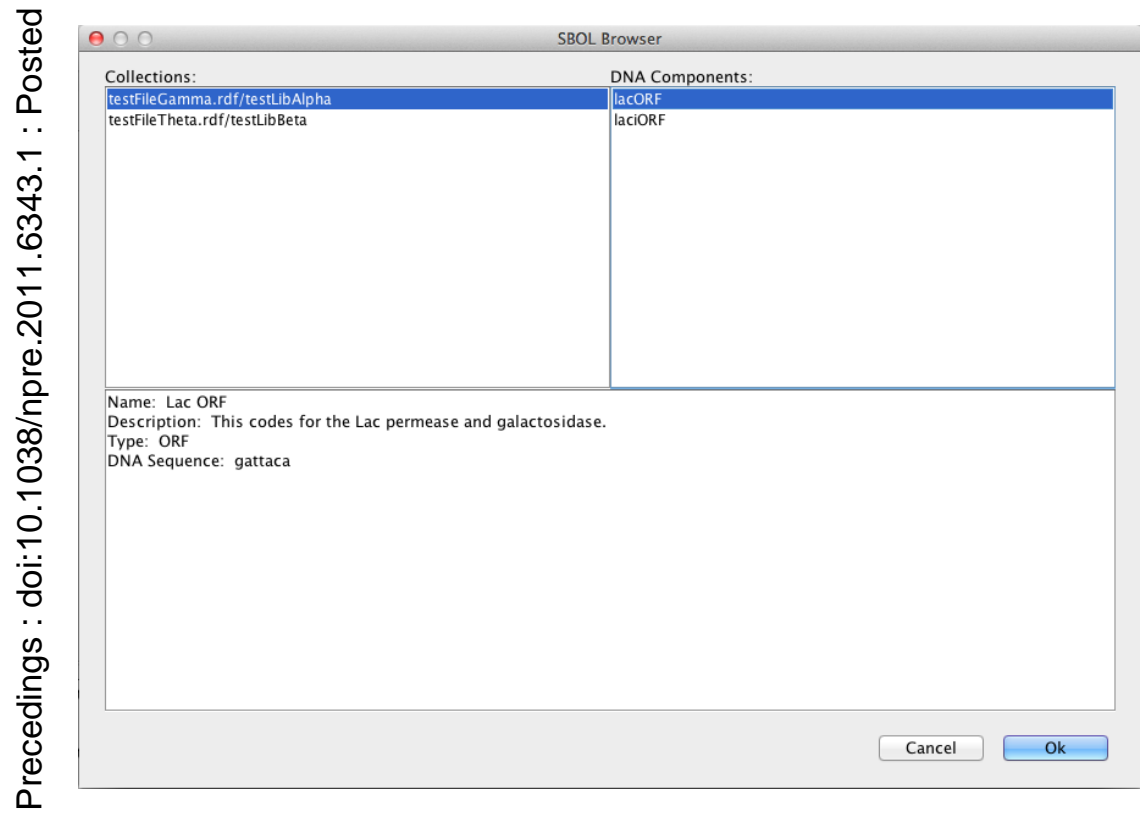




\section{SBOL Extensions}

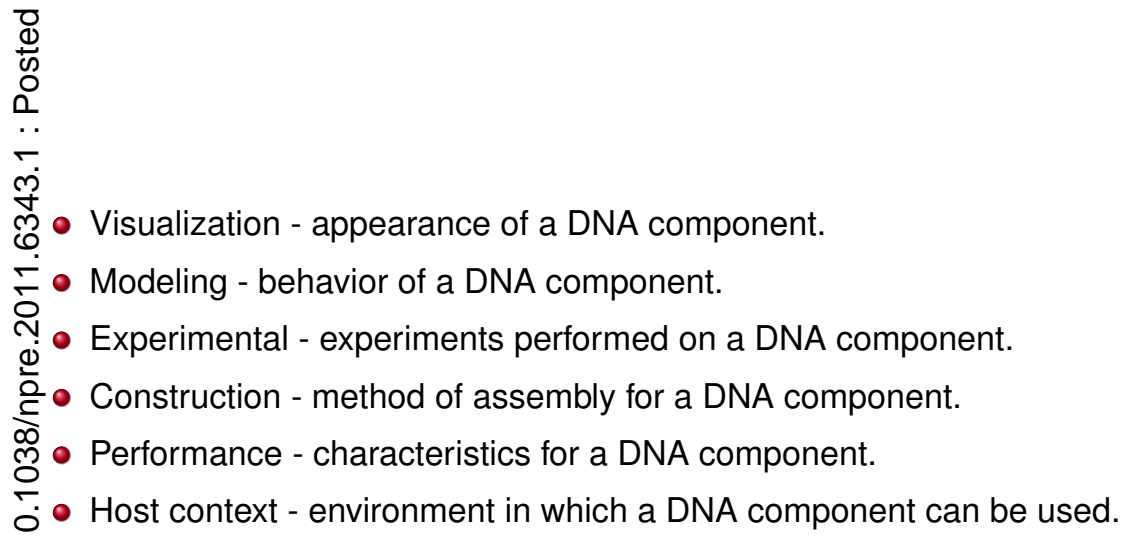




\title{
SBOL Visual Model
}
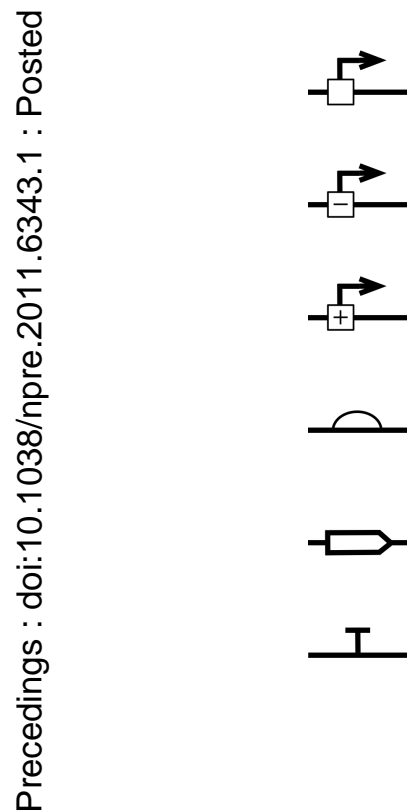

Forward Constitutive Promoter

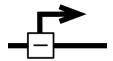

Forward Repressible Promoter

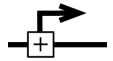

Forward Inducible Promoter

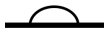

Forward Translation Start Site

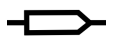

Forward Open Reading Frame

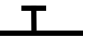

\author{
Forward Terminator \\ etc.
}




\section{SBOL Modeling Extension (Preliminary)}

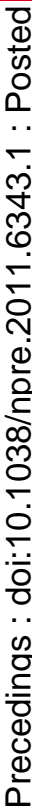

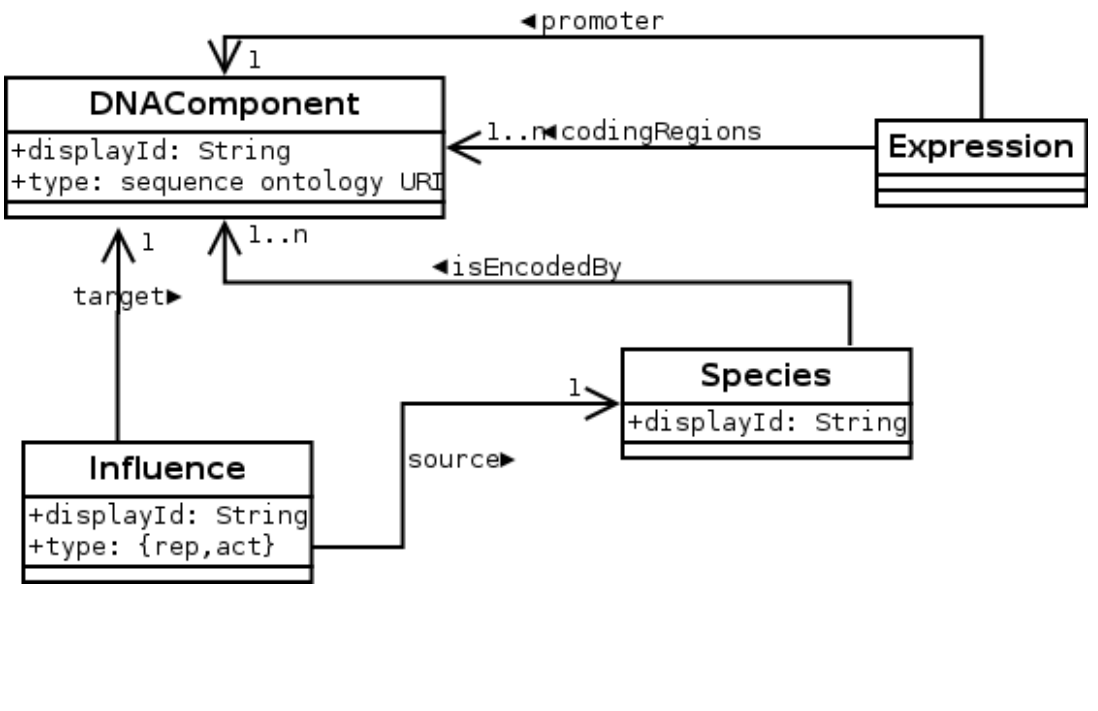




\section{Discussion}

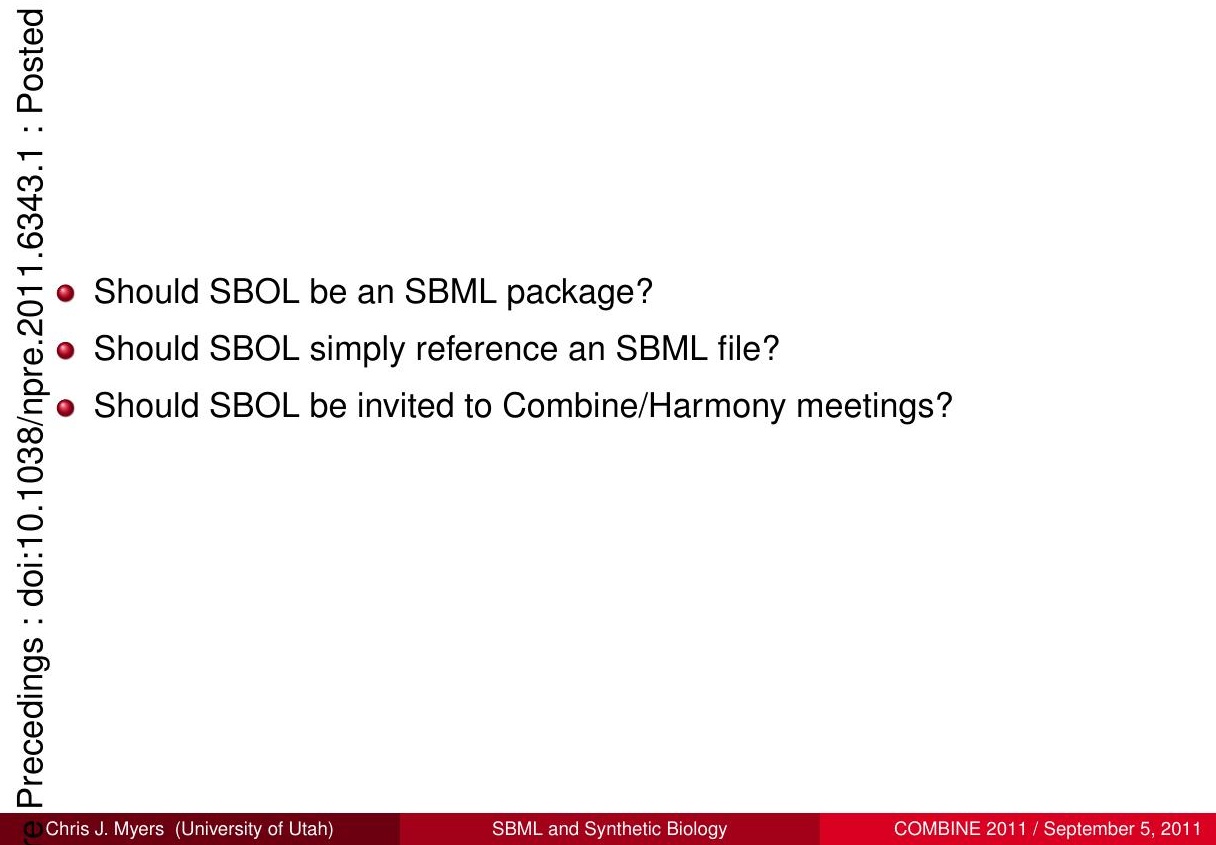

